\title{
Itinerário terapêutico de pacientes com deficiência auditiva
}

\author{
Therapeutic itinerary of hearing \\ impaired patients
}

\section{Itinerario terapéutico de pacientes con discapacidad auditiva}

\author{
Kenia Isabella de Souza* (i) \\ Camila Ferreira Ambrosio* \\ Teresa Maria Momensohn Santos** (i)
}

\begin{abstract}
Resumo
Introdução: As ações do serviço de saúde auditiva são executadas pela atenção básica, média e alta complexidade de maneira hierarquizada, organizada para facilitar o diagnóstico, recuperação e reabilitação. Conhecer o itinerário percorrido pelo usuário pode influenciar a solução adotada e identificar dificuldades ao diagnóstico precoce. Objetivo: Analisar o itinerário terapêutico e perfil dos pacientes diagnosticados com deficiência auditiva de um serviço de Saúde Auditiva Microrregional de Minas Gerais. Método: Análise descritiva retrospectiva dos dados de prontuários do serviço auditivo de Sete Lagoas, de 189 sujeitos que receberam aparelho auditivo pelo Serviço Único de Saúde entre os anos de 2018/2019 no serviço de referência em Média Complexidade da Microrregião. Observa-se maior número de usuários com idade superior a 60 anos em Sete Lagoas e também na microrregião. A classificação quanto a sexo demonstra predomínio de mulheres em toda área de atendimento, e, principalmente, no município de Sete Lagoas. Resultados: A análise do itinerário mostra que o tempo entre a chegada ao serviço de saúde auditiva e as etapas de pré molde, teste e entrega do aparelho auditivo é menor para crianças do que para adultos e idosos. Conclusão: O itinerário percorrido pelos pacientes interfere em maior aproveitamento do aparelho auditivo e adaptação adequada devido ao tempo de espera e distância percorridos. Adultos e idosos têm maior itinerário em relação às crianças.
\end{abstract}

Palavras-chave: Perda Auditiva; Acesso aos Serviços de Saúde; Saúde Pública.

\footnotetext{
* IEAA - Instituto de Estudos Avançados em Audição - São Paulo (SP), Brasil.

** Pontifícia Universidade Católica de São Paulo - PUC-SP- São Paulo (SP), Brasil.
}

Contribuição dos autores:

KIS, CFA: Planejamento da pesquisa, coleta dos dados, elaboração do relatório final. TMMS: Orientação e supervisão do projeto; correção do relatório final.

E-mail para correspondência: Kenia Isabella de Souza - keniaisa@yahoo.com.br Recebido: 16/07/2020

Aprovado: 11/12/2020 


\section{Abstract}

Introduction: The actions of the hearing health service are performed by basic, middle and high complexity care in a hierarchical manner, organized to facilitate diagnosis, recovery and rehabilitation. Knowing the route taken by the user can influence the solution adopted and identify difficulties to early diagnosis. Objective: To analyze the profile and therapeutic itinerary of patients diagnosed with hearing impairment in a Microregional Hearing Health Service in Minas Gerais. Method: Retrospective descriptive analysis of the medical records data from the hearing service Sete Lagoas, of 189 subjects who received hearing aids by the Unified Health Service between the years 2018/2019 at the reference service on Medium Complexity in the Microregion. There are a greater number of users over the age of 60 in Sete Lagoas and also in the micro-region. The classification as to sex shows a predominance of women in the entire service area and mainly in the city of Sete Lagoas. Results: The analysis of the itinerary shows that the time between arrival at the hearing health service and the stages of pre-mold, test and delivery of the hearing aid is shorter for children than for adults and the elderly. A higher number of users over 60 years of age is observed in Sete Lagoas and also in the microregion. Conclusion: The route taken by the patients interferes with the use of the hearing aid and a proper adaptation due to the waiting time and distance covered. Adults and elderly have a longer itinerary in relation to children.

Keywords: Hearing Loss; Health Services Accessibility; Public Health.

\section{Resumen}

Introducción: Las acciones del servicio de salud auditiva son realizadas por atención primaria, media y alta complejidad de manera jerárquica, organizadas para facilitar el diagnóstico, la recuperación y la rehabilitación. Conocer la ruta tomada por el usuario puede influir en la solución adoptada y identificar dificultades en el diagnóstico temprano. Objetivo: analizar el perfil y el itinerário y perfil terapéutico de pacientes diagnosticados con discapacidad auditiva en un servicio de salud auditiva microrregional en Minas Gerais. Método: Análisis descriptivo retrospectivo de los datos de las historias clínicas del servicio de audición en Sete Lagoas, de 189 sujetos que recibieron audífonos por parte del Servicio Unificado de Salud entre los años 2018/2019 en el servicio de referencia de Complejidad Media en la Microrregión. Hay un mayor número de usuarios mayores de 60 años en Sete Lagoas y también en la microrregión. La clasificación por sexo muestra un predominio de mujeres en toda el área de servicio y principalmente en la ciudad de Sete Lagoas. Resultados: El análisis del itinerario muestra que el tiempo entre la llegada al servicio de salud auditiva y las etapas de premoldeo, prueba y entrega del audífono es más corto para niños que para adultos y ancianos. Se observa un mayor número de usuarios mayores de 60 años en Sete Lagoas y también en la microrregión. Conclusión: el itinerario recorrido por los pacientes interfiere con un mayor uso del audífono y una adaptación adecuada debido al tiempo de espera y la distancia recorrida. Los adultos y los ancianos tienen un itinerario mayor que los niños.

Palabras clave: Pérdida Auditiva; Accessilbilidad a los Servicios de Salud; Salud Pública.

\section{Introdução}

A Política Nacional de Atenção à Saúde Auditiva (PNASA) foi instituída pelo Ministério da Saúde para execução das ações em saúde auditiva de forma hierarquizada, regionalizada e integrada entre a atenção básica, média e a de alta complexidade, para garantir o diagnóstico, promoção, proteção e reabilitação auditiva no Serviço Único de Saúde (SUS) ${ }^{1}$.
Em outubro de 2004, as Portarias MS/SAS 587 e MS/SAS 589 constituíram o credenciamento e as atribuições dos diferentes níveis de complexidade dos Serviços de Saúde Auditiva. A Atenção Básica é responsável por ações coletivas ou individuais de promoção e prevenção da saúde auditiva com atividades educativas e informativas bem como identificar deficiência auditiva de forma precoce. O diagnóstico e terapia fonoaudiológica são responsabilidade do serviço de média complexidade 
o qual atende indivíduos com risco ou suspeita de perda auditiva e com a deficiência auditiva já diagnosticada, proporcionando estrutura física e profissional apropriadas ao atendimento. O serviço de média complexidade não atende crianças com idade inferior a três anos, além de triagem e monitoramento da audição de neonatos e escolares. A alta complexidade, por sua vez, abrange a avaliação e diagnóstico de crianças com menos de três anos de idade e de pacientes com afecções associadas de qualquer natureza além de realizar os mesmos serviços da média complexidade com uma equipe multiprofissional maior ${ }^{2}$.

Para ampliar a cobertura assistencial proporcional à população estimada do Brasil em 2004, de 169.872.856 habitantes, foi deliberado o número mínimo de 116 estabelecimentos de saúde auditiva de média e alta complexidade em todo o país, assim distribuídos: 16 na região Sul, 47 no Sudeste, 9 no Centro-Oeste, 33 na região Nordeste e 11 no Norte do país ${ }^{1,3}$.

A Secretaria de Estado de Minas Gerais implantou a Rede de Atenção à Saúde Auditiva regulado na PNASA, para garantir o atendimento integral ao indivíduo com deficiência auditiva; em 2009 havia 12 serviços de Saúde Auditiva credenciados, sete de alta complexidade e cinco de média complexidade. Além dos Serviços de Atenção à Saúde Auditiva existem outros pontos de atenção: Fonoaudiologia Descentralizada (Secretaria Municipal de Saúde/Atenção primária), Junta de Saúde Auditiva Microrregional, Junta Reguladora da Saúde Auditiva, Serviços de Referência de Triagem Auditiva Neonatal e Serviços de Implante Coclear ${ }^{4}$.

Os usuários com alterações auditivas procuram os serviços de saúde auditiva em busca de melhora da sua comunicação e da sua função auditiva. A efetividade do tratamento de adaptação de Aparelho de Amplificação Sonora Individual e/ ou reabilitação auditiva precisa ser analisada por todos os ângulos ${ }^{5,6}$.

A trajetória destas pessoas à procura da manutenção ou recuperação da saúde é denominada itinerário terapêutico, o qual incorpora recursos variados de uma rede assistencial organizada ${ }^{7,8}$.

Conhecer o itinerário percorrido pelo paciente permite a identificação de fatos que podem dificultar o diagnóstico precoce e o processo terapêutico de pacientes em todos os aspectos ${ }^{9}$.
Desta forma, o presente estudo teve como objetivo analisar o itinerário terapêutico e perfil dos pacientes diagnosticados com deficiência auditiva de um serviço de Saúde Auditiva Microrregional de Minas Gerais.

\section{Métodos}

Trata-se de uma análise descritiva retrospectiva dos dados de prontuários constantes na Unidade de Saúde Auditiva do município de Sete Lagoas, dos sujeitos que receberam aparelho auditivo pelo SUS nos anos de 2018 e 2019 no serviço de referência em Média Complexidade da Microrregião de Sete Lagoas. O estudo consistiu em análise documental dos prontuários considerando evoluções de avaliações, exames, marcações de atendimento e reuniões registradas de cada paciente candidato a aparelho auditivo pelo SUS do município de Sete Lagoas e microrregião.

A amostra analisada foi composta por 189 indivíduos, $150(79,4 \%)$ residentes do município de Sete Lagoas e 39 (20,6\%) pertencentes a outros 23 municípios da microrregião atendida.

A análise dos dados coletados foi realizada pela estatística percentual e apresentada em gráficos e tabelas para facilitação da apresentação dos possíveis resultados.

O projeto foi submetido ao Comitê de Ética e Pesquisa e aprovado sob número 3.984.685, com dispensa do Termo de Consentimento Livre e Esclarecido considerando o compromisso ético dos pesquisadores envolvidos e a utilização somente de dados documentais referentes aos atendimentos habituais da Unidade de Saúde de Sete Lagoas. O sigilo de nomes dos pacientes será mantido, e, se necessário, serão utilizadas siglas fictícias.

A Unidade de Saúde Auditiva de Sete Lagoas recebe os pacientes encaminhados pelas Unidades Básicas de Saúde do município e Microrregião com queixas relacionadas à audição. A referência em Média Complexidade para pacientes desta microrregional é situada no município de Santa Luzia em Minas Gerais.

O município de Sete Lagoas apresenta uma população de 239.639 habitantes, e a microrregião atendida pela Saúde Auditiva da cidade, composta por 23 municípios com raio de até $222 \mathrm{~km}$, soma 209.433 habitantes ${ }^{10,11}$. Para atendimento de Baixa Complexidade (município de Sete Lagoas), os pacientes de outros municípios podem percorrer 
distâncias aproximadas de $12 \mathrm{~km}$ até $222 \mathrm{~km}$. Para os atendimentos realizados em Média Complexidade (município de Santa Luzia) a distância percorrida pelos mesmos pacientes pode variar de $53 \mathrm{~km}$ a $294 \mathrm{~km}$.

A Figura 1 mostra o processo de atendimento dos pacientes residentes do próprio município. Ocorre o atendimento com profissional especia- lista, avaliação audiológica básica e diagnóstico na Unidade de Saúde Auditiva. O paciente pode optar pela avaliação audiológica básica em serviço privado e o processo pode ser alterado. Quando necessária, a adaptação de aparelho auditivo ocorre no serviço de referência em média complexidade em duas consultas.

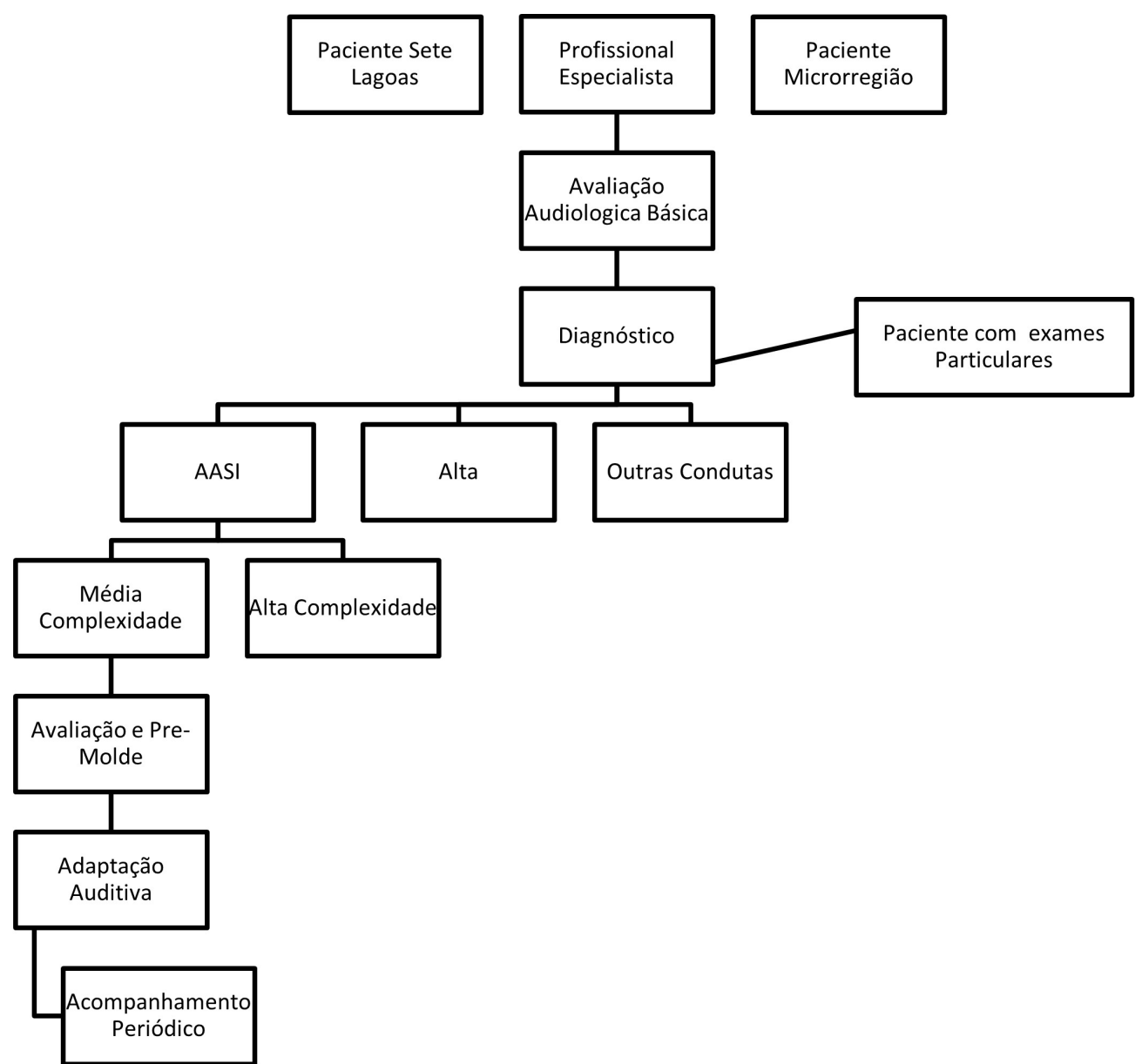

Figura 1. Fluxograma do atendimento no serviço auditivo em Sete Lagoas e Microrregião

Todas as etapas seguidas pelos candidatos a Aparelho de Amplificação Sonora Individual (AASI) são registradas em prontuários guardados na própria unidade, desde que tenham realizado a avaliação audiológica básica no serviço público, caso contrário só entra no sistema de regulação de vagas para agendar a data de pré-molde e adaptação de Aparelho. 
Tabela 1. Distribuição dos pacientes encaminhados para avaliação de AASI pela Unidade de Saúde Auditiva de Sete Lagoas de janeiro de 2018 a dezembro de 2019.

\begin{tabular}{|c|c|c|c|c|c|c|}
\hline \multirow{2}{*}{$\begin{array}{l}\text { Região de } \\
\text { origem }\end{array}$} & \multirow{2}{*}{$\begin{array}{c}\text { Número de } \\
\text { indivíduos } \\
\text { avaliados } \\
\text { para } \\
\text { concessão de } \\
\text { AASI } \\
\end{array}$} & \multicolumn{2}{|c|}{ Sexo } & \multirow{2}{*}{$\begin{array}{l}\text { Indivíduos } \\
\text { com idade de } \\
3 \text { a } 18 \text { anos }\end{array}$} & \multirow{2}{*}{$\begin{array}{l}\text { Indivíduos } \\
\text { com idade de } \\
19 \text { a } 59 \text { anos }\end{array}$} & \multirow{2}{*}{$\begin{array}{c}\text { Indivíduos } \\
\text { com idade } \\
\text { acima de } 60 \\
\text { anos }\end{array}$} \\
\hline & & Masculino & Feminino & & & \\
\hline & & n (\%) & n (\%) & n (\%) & n (\%) & n (\%) \\
\hline Sete Lagoas & 150 & $66(77,6)$ & $84(80,8)$ & $6(54,5)$ & $50(78,1)$ & $94(82,5)$ \\
\hline Microrregião & 39 & $19(22,4)$ & $20(19,2)$ & $5(45,5)$ & $14(21,9)$ & $20(17,5)$ \\
\hline TOTAL & 189 & $85(100,0)$ & $104(100)$ & $11(100,0)$ & $64(100,0)$ & $114(100,0)$ \\
\hline
\end{tabular}

Conforme apresentado na Figura 2, o tempo de espera total dos pacientes desde o encaminhamento emitido pela Unidade Básica de Saúde até a adaptação do AASI em média complexidade superior a 60 anos em Sete Lagoas e também na microrregião. A classificação quanto a sexo demonstra predomínio de mulheres em toda área de atendimento e principalmente no município de Sete Lagoas.

Observa-se maior número de usuários com idade

mostra uma espera maior para idosos do município de Sete Lagoas, o qual é proporcional à quantidade de idosos com indicação para avaliação de aparelho auditivo.

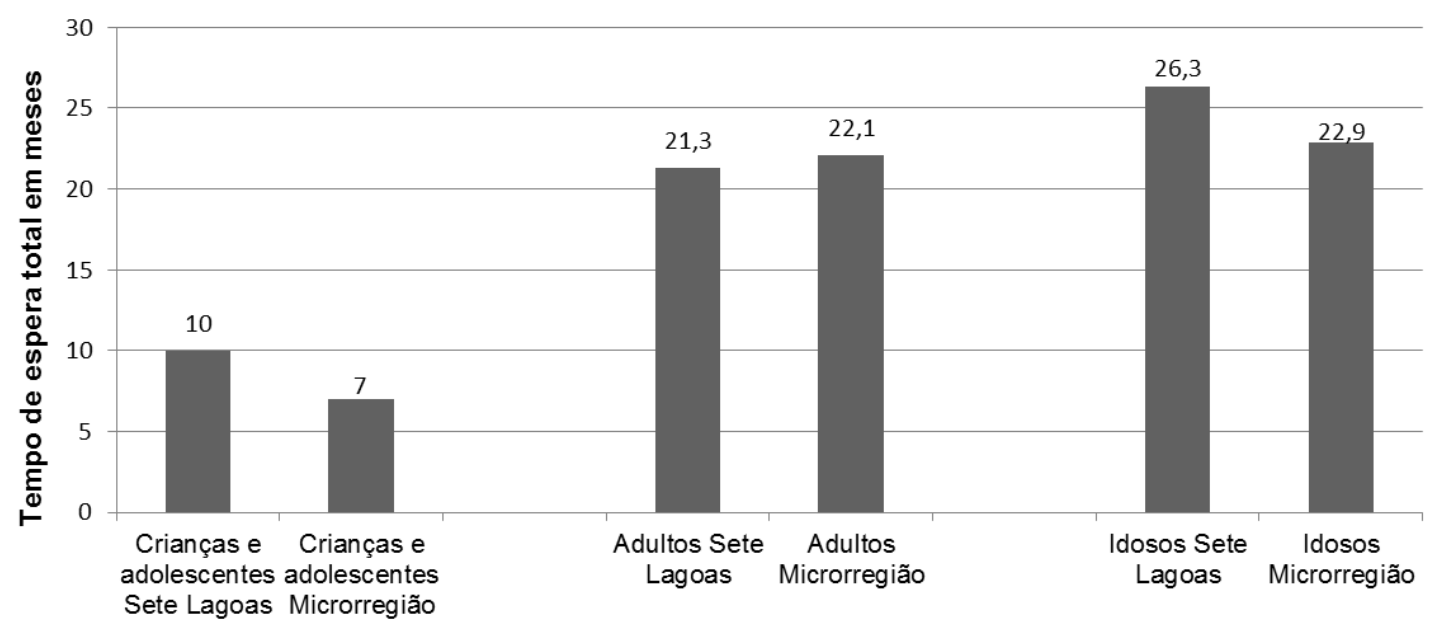

Figura 2. Tempo de espera total em meses, que os pacientes percorrem desde o encaminhamento até a adaptação de AASI. 
As etapas específicas de espera de residentes do município de Sete Lagoas são descritas com suas respectivas durações na Tabela 2, observa-se que a etapa de maior permanência é a de primeiro contato com o serviço, na qual a atenção básica realiza o agendamento para atendimento na Unidade de Saúde Auditiva. Deve-se considerar um período de ausência de audiômetro no ano de 2017 para assistência técnica.

Tabela 2. Tempo de espera (em meses) das etapas de atendimentos na Unidade de Saúde Auditiva de Sete Lagoas segundo a faixa etária do paciente.

\begin{tabular}{lccc}
\hline Etapa & Crianças (meses) & Adultos (meses) & Idosos (meses) \\
\hline Primeiro contato com o serviço & 6,3 & 7,8 & 6,5 \\
Obter diagnóstico & 0 & 1,9 & 3,1 \\
Diagnóstico à primeira avaliação & 2 & 10,3 & 14.9 \\
Primeira avaliação à busca de AASI & 1,7 & 1,3 & 1,8 \\
\hline
\end{tabular}

A Tabela 3 mostra a quantidade de pacientes de Sete Lagoas que retornaram para orientação, acompanhamento ou conserto nos serviços de baixa ou média complexidade. Os 150 pacientes do município deveriam ter voltado ao serviço de baixa complexidade para a orientação e contra referência após a adaptação, 128 deveriam ter comparecido ao serviço de média complexidade para o acompanhamento anual, considerando que 22 ainda não completaram 1 ano de busca de aparelho até a data de análise dos dados. É possível notar que o número de pacientes que buscam o serviço após a adaptação do Aparelho auditivo é bem menor que o esperado, tanto no serviço de baixa complexidade como no serviço de média complexidade. Todas as crianças atendidas retornam para o serviço de baixa complexidade. A comparação mostra que alguns adultos voltam ao serviço de média complexidade diretamente sem contato com o serviço de baixa complexidade, e os idosos apresentam-se mais ao serviço do próprio município de residência que o de média complexidade.

Tabela 3. Número e perfil por idade de indivíduos de Sete Lagoas que receberam os AASI's e retornaram ao serviço de baixa ou média complexidade para atendimento

\begin{tabular}{lccccc}
\hline Serviço & Expectativa & Retornaram & $\begin{array}{c}\text { Crianças e } \\
\text { adolescentes }\end{array}$ & Adultos & Idosos \\
\hline Baixa Complexidade & 150 & 63 & 3 & 19 & 41 \\
Média Complexidade & 128 & 51 & 2 & 23 & 26 \\
\hline
\end{tabular}

\section{Discussão}

A análise do perfil atendido pela Unidade de Saúde Auditiva de Sete Lagoas aponta maioria de indivíduos residentes no próprio polo por ser critério do estudo a realização de todas as etapas dentro do serviço de saúde pública.

O número expressivo de idosos da amostra está em similaridade com estudos anteriores ${ }^{12-14} \mathrm{e}$ pode ser relacionado a fatores como a perda relacionada à idade. Existe uma baixa representatividade de crianças compatível com outro estudo por se tratar de um serviço de média complexidade ${ }^{12}$. Em relação a gênero, a quantidade superior de mulheres contrasta com números encontrados fora do país ${ }^{15}$, porém no Brasil existe uma maior preocupação apresentada pelo gênero feminino, relacionada aos cuidados à saúde em geral bem como com a perda auditiva ${ }^{16}$.

Não houve diferença quanto ao tempo de espera entre adultos e idosos. As crianças estão entre as prioridades clínicas da unidade, assim o atendimento é realizado de maneira mais ágil. A espera total até a adaptação de aparelho demonstra-se superior ao tempo encontrado em outro estudo 
que indica demora de 3 a 6 meses no processo ${ }^{17}$. É importante considerar fatores que podem intervir no tempo do processo como a efetividade do serviço de referência e contra referência, ampliação do serviço de diagnóstico e a necessidade de aumento do número de concessão de aparelhos auditivos por existir progressão da demanda com perda auditiva devido à longevidade populacional, entre outros fatores causais. As microrregiões foram planejadas para que atendam áreas com 1.500.000 habitantes. Inicialmente, esta proporção pareceu ser adequada; com o decorrer dos anos, o que se observou é que o crescimento da população atendida nos serviços de saúde ocorre de forma geométrica - para cada paciente novo que é inscrito no programa de uma instituição, se somam os pacientes que devem retornar após um mês, outro que retorna em três meses, outro que deve voltar em um ano. Além destes, temos os que procuram o serviço para trocar o molde, para repor aparelho perdido, quebrado ou que já não atende à sua demanda auditiva.

A microrregião de Sete Lagoas é composta por muitos municípios e possui uma população numerosa. A distância percorrida para o atendimento, seja no município de Sete Lagoas ou em Santa Luzia é extensa, e este deslocamento pode interferir no uso efetivo do aparelho auditivo visto a dificuldade de percurso até mesmo para reparos simples. Alguns municípios não possuem fonoaudiólogos descentralizados e não há fornecimento de recursos para atendimentos básicos na baixa complexidade, como trocas de tubos de AASI's e baterias para teste.

O serviço descentralizado solicita ao paciente retorno após o recebimento do aparelho para orientações, oferece terapia fonoaudiológica de reabilitação auditiva e grupos para auxiliar a adaptação. No entanto, o baixo número de pacientes que retornam ao atendimento periódico propõe a reflexão quanto ao aproveitamento eficaz dos aparelhos distribuídos, pois o acompanhamento é de fundamental importância, tanto para o uso efetivo quanto para assegurar uma adaptação adequada ${ }^{18}$.

Entre os pacientes de retorno analisados, apenas 11 manifestaram espontaneamente o motivo de não comparecimento à média complexidade conforme registros durante o atendimento na Unidade de Saúde de Sete Lagoas. Nota-se que os motivos de saúde são considerados os maiores empecilhos para o deslocamento até o município de Santa Luzia. Os problemas relatados pelos pacientes coin- cidem com determinados apontados pela literatura como a dificuldade de acesso ao serviço, problemas familiares e pessoais, adaptação apropriada ou não uso do aparelho ${ }^{19-21}$

A necessidade maior que a demanda de aparelhos auditivos ofertados é uma realidade enfrentada não somente pelo Brasil ${ }^{22}$. Diante da considerável fila de espera, da ampla população atendida e dos empecilhos citados para o acompanhamento periódico ativo, uma maior oferta de serviços voltados ao atendimento de saúde auditiva deve ser avaliada para a atenção integral ao paciente com perda auditiva conforme o preconizado pela PNASA ${ }^{1}$.

O processo de adaptação de aparelhos poderia se tornar mais personalizado e priorizar as demandas do usuário. Facilitar o acesso ao atendimento e credenciamento de novos serviços de média complexidade em Saúde Auditiva mais próximos aos usuários torna-se uma solução viável, de forma que os serviços não fiquem concentrados apenas nos grandes municípios por demandar um complexo deslocamento, e nem sempre o paciente estar disposto a realizá-lo.

\section{Conclusão}

O itinerário percorrido pelos pacientes com deficiência auditiva interfere em um maior aproveitamento do aparelho auditivo e na adaptação adequada devido ao tempo de espera e à distância percorrida. Após este estudo, constatamos que adultos e idosos têm um tempo maior no itinerário ao processo de adaptação de aparelho em relação às crianças. As crianças de Sete Lagoas demoram mais que as crianças da Microrregião para concluir o processo.

Uma das maiores queixas levantadas pelos usuários é a distância percorrida para chegar ao serviço de concessão de aparelho, e muitas vezes deixam de realizar as manutenções necessárias para evitar o deslocamento.

\section{Referências}

1. Ministério da Saúde. Portaria n ${ }^{\circ} 2.073$, de 28 de setembro de 2004. Brasília: Ministério da Saúde; out 2004.

2. Ministério da Saúde. Saúde auditiva. Portarias de saúde auditiva. Brasília: Ministério da Saúde; out 2004.

3. Teixeira CF. Estudo avaliativo da política de atenção à saúde auditiva: estudo de caso em Pernambuco [tese]. Recife: Centro de Pesquisa Aggeu Magalhães, Fundação Oswaldo Cruz; 2007. 
4. Maciel FJ, Januário GC, Henriques CMA, Esteves CC, Silva MA, Carvalho SAS, Lemos SMA ACR. Indicadores de saúde auditiva em Minas Gerais - um estudo por macrorregião. 2013; 18(4): 275-84.

5. Cook JA, Hawkins DB. Outcome measurement for patients receiving hearing aid services. Laryngoscope. 2007; 117(4): 610-3.

6. Tamaki EM, Tanaka OY, Felisberto E, Alves CKA, Drumond Junior M, Bezerra LCA, et al. Metodologia de construção de um painel de indicadores para o monitoramento e avaliação da gestão do SUS. Ciênc Saúde Colet. 2012; 17(4): 839-494.

7. Brustolin AFF. Therapeutic itinerary of elderly cancer survivors. Acta paul. enferm. 2017; 30(1): -59.

8. Pinho PA, Pereira PPG. Therapeutic itineraries: paths crossed in the search for care. Interface - Comunic, Saude, Educ. 2012; 16(41): 435-47.

9. Aquino RCA, Lima MLLT, Silva VL, Alencar FL, Rodrigues $M$. Therapeutic itinerary and access to health services in cases oral cancer deaths. Rev. CEFAC. 2018 Set-Out; 20(5): 595-603.

10. Instituto Brasileiro de Geografia e Estatística (página da internet), Diretoria de Pesquisas, Coordenação de População e Indicadores Sociais, Estimativas da população residente com data de referência 01 de julho de 2019. Disponível em: https:// www.ibge.gov.br/institucional.

11. Rota Mapas. (página da internet). Acesso em 06 de maio de 2020. Disponível em: https://www.rotamapas.com.br/minasgerais. Brasil

12. Gresele, ADP, Lessa AH, Alves LC, Torres EMO, Vaucher AVA, Moraes AB, Costa MJ. Levantamento e análise de dados de pacientes atendidos em um programa de concessão de aparelhos de amplificação sonora individual. CoDAS 2013; 25(3): 195-201.

13. Chien W, Lin FR. Prevalence of Hearing Aid Use among Older Adults in the United States. Arch Intern Med. 2012; 172(3): 292-3.

14. Correia RO, Pinheiro CCD, Paiva FCG, Gomes-Neto PS, Rodrigues TP, Mendonça ATB, Freitas MR. Reabilitação auditiva por aparelhos de amplificação sonora individual (AASI): perfil epidemiológico de pacientes adaptados em um hospital terciário em 5 anos. Rev Med UFC. 2017; 57(2): 26-30.

15. Roth TN, Hanebuth D, Probst R. Prevalence of age-related hearing loss in Europe: a review. Eur Arch Otorhinolaryngol. 2011; 268(8): 1101-7.

16. Espmark AK, Rosenhall U, Erlandsson S, Steen B. The two faces of presbyacusis: hearing impairment and psychosocial consequences. Int J Audiol. 2002; 41(2): 125-35.

17. Jardim, D S; Maciel, F J; Lemos, S MA. Perfil epidemiológico de uma população com deficiência auditiva. Rev. CEFAC. 2016 Maio-Jun; 18(3): 746-57.

18. Ribas A; Rosa M; Dante G; Martins-Basseto, J. SOS Prótese Auditiva - relato de um estudo de caso realizado na Clínica de Fonoaudiologia da UTP. Tuiuti: Ciência e Cultura, n. 38, p. 09-18, Curitiba, 2006.

19. Popelka, M. M., Cruickshanks, K. J., Wiley, T. L., Tweed, T. S., Klein, B. E. K., \& Klein, R. (1998). Low prevalence of hearing aid use among older adults with hearing loss: The epidemiology of hearing loss study. Journal of the American Geriatrics Society, 46(9), 1075-8.
20. Freitas CD, Costa MJ. Processo de adaptação de próteses auditivas em usuários atendidos em uma instituição pública federal - parte I: resultados e implicações com o uso da amplificação. Rev Bras Otorrinolaringol 2007; 73(6): 744-51.

21. Abby McCormack \& Heather Fortnum (2013) Why do people fitted with hearing aids not wear them. Int J Audiol. 52: $5,360-8$.

22. Vera-Genevey H, Lebogang R. Rehabilitation for disabling hearing loss: evaluating the need relative to provision of hearing aids in the public health care system, Disability and Rehabilitation, 41: 22, 2704-7. 